\title{
Surface and Cytoplasmic Immunoglobulin Expression in Circulating B-Lymphocytes in Acute Kawasaki Disease
}

\author{
DELANE SHINGADIA, MAURICE O'GORMAN, ANNE H. ROWLEY, AND \\ STANFORD T. SHULMAN \\ Divisions of Infectious Diseases [D.S., A.H.R., S.T.S.] and Immunology [M.O.], Department of Pediatrics, \\ Children's Memorial Hospital/Northwestern University, Chicago IL 60614
}

\begin{abstract}
Kawasaki disease (KD) is an acute vasculitis of young childhood predominantly affecting the coronary arteries. IgA plasma cells have been found to infiltrate vascular and nonvascular tissues in fatal acute KD. To determine whether IgA Blymphocytes were increased in the peripheral blood of patients with $\mathrm{KD}$, we performed three-color flow cytometry to detect surface and cytoplasmic immunoglobulin expression (IgA, IgM, $\mathrm{IgD}$, and $\operatorname{IgG}$ ) of peripheral B-lymphocytes in $\mathrm{KD}$ patients during the acute, subacute, and convalescent stages of illness and in age-matched febrile and afebrile pediatric controls. Surprisingly, absolute numbers of B-lymphocytes expressing IgA were found to be significantly lower in peripheral blood of acute $\mathrm{KD}$
\end{abstract}

\section{ABSTRACT}

patients compared with febrile and afebrile pediatric controls. These findings indicate that IgA plasma cells are not present in $\mathrm{KD}$ tissue as a result of excess numbers of these $\operatorname{IgA} \mathrm{B}$ lymphocytes in peripheral blood. We speculate that IgA Blymphocytes are selectively withdrawn from the peripheral circulation into KD target tissues as part of a specific IgA immune response. (Pediatr Res 50: 538-543, 2001)

\section{Abbreviations:}

IVGG, i.v. gamma globulin

KD, Kawasaki disease

NK, natural killer
$\mathrm{KD}$ is an acute vasculitis of young childhood that affects the coronary arteries. It is the commonest cause of acquired heart disease in the United States and Japan (1). Both the clinical features and epidemiology of KD suggest an infectious etiology although the causative agent remains unknown. IVGG therapy has been shown to reduce inflammatory signs and to prevent coronary artery abnormalities that occur in $25 \%$ of untreated patients $(2,3)$.

The novel finding that IgA plasma cells infiltrate vascular tissue, including coronary arterial walls, has been reported in children with fatal KD (4). Analysis of IgA clonality in vascular tissue from all of three acute fatal KD cases indicated an oligoclonal, antigen-driven immune response (5). Further study indicated that IgA plasma cells also infiltrate nonvascular tissues, such as the respiratory tract, kidney, and pancreas in acute KD (6). In particular, IgA plasma cells were significantly increased in the trachea of children who had died of acute KD, similar to findings in children with fatal respiratory viral infections. These data strongly support entry of the KD etio-

Received December 22, 2000; accepted June 18, 2001.

Correspondence and reprint requests: Delane Shingadia, Flat 3, 120 Goldhurst Terrace, London NW6 3HR, England; e-mail: d.v.shingadia@mds.qmw.ac.uk

Supported by the Kawasaki Disease Research Fund, Children's Memorial Hospital, Chicago. logic agent through the upper respiratory tract, resulting in a specific mucosal IgA immune response, with subsequent systemic spread to vascular tissue, pancreas, and kidney.

IgA plasma cell infiltration into vascular tissue in $\mathrm{KD}$ seems unusual because $\operatorname{IgA}+$ B-lymphocytes account for only 2 to $7 \%$ of B-lymphocytes in the peripheral blood of adults, which themselves represent only 10 to $31 \%$ of the total peripheral blood lymphocyte population (7). The presence of these cells in vascular tissue could be the result of a large increase in circulating $\operatorname{IgA}+\mathrm{B}$-lymphocytes in acute $\mathrm{KD}$ as part of a nonspecific inflammatory response. If this were the case, a large increase in IgA + B-lymphocytes in the peripheral blood in acute $\mathrm{KD}$ might serve as a diagnostic marker of the illness. Alternatively, IgA + B-lymphocytes could be selectively withdrawn from the peripheral blood into vascular tissue as part of a specific immune response in $\mathrm{KD}$.

Circulating B-lymphocytes are not a single, homogenous population but instead can be divided into several subpopulations, reflecting different stages in their maturation. The final stage of this maturation process is the terminal differentiation into antibody-secreting plasma cells, which is characterized by irreversible alterations in the expression of surface markers, such as immunoglobulin, CD20, and CD38 antigens. Whereas nonterminally differentiated B-lymphocytes express surface 
immunoglobulin and CD20 antigen, plasma cells lose these markers and instead express surface CD38 antigen and cytoplasmic immunoglobulin. There is, however, a great deal of heterogeneity in this process with variability of immunoglobulin and surface antigen expression (8). To account for this heterogeneity and also to ensure that we detected all IgA expressing cells in peripheral blood, we used a flow cytometric technique to determine surface and cytoplasmic immunoglobulin expression (IgA, IgM, IgD, and $\operatorname{IgG}$ ) of circulating Blymphocytes in acute, subacute, and convalescent KD (before and after IVGG therapy), and in febrile and afebrile age- and sex-matched children

\section{METHODS}

Participants were enrolled from Children's Memorial Hospital, Chicago, from September 1998 to August 1999. Children with acute $\mathrm{KD}$ were febrile for $>4 \mathrm{~d}$ and manifested at least four of the following findings on examination: a polymorphous generalized erythematous rash, extremity changes consisting of induration of the hands and feet with erythematous palms and soles, bilateral nonexudative conjunctival injection, erythematous mouth and pharynx with red cracked lips and strawberry tongue, and acute nonpurulent cervical adenopathy. Blood samples were obtained from KD patients before receiving IVGG and at follow-up ( 2 and 8 wk after IVGG). For each subject with acute $\mathrm{KD}$, an age- and sex-matched febrile and nonfebrile control patient was enrolled as soon as possible after diagnosis of the index KD patient. Febrile controls (children with fever $>38.5^{\circ} \mathrm{C}$ for $>2 \mathrm{~d}$ duration and with an infectious etiology) were recruited from the emergency room and inpatient units. Nonfebrile controls were children who were undergoing elective surgery. Children with underlying immunodeficiency, including HIV infection, were excluded from both control groups. Age-matched control patients were chosen using the following age ranges: $<6 \mathrm{mo} \pm 2 \mathrm{mo} ; 6-12 \mathrm{mo} \pm$ 4 mo; 12 mo- 4 y \pm 6 mo; $>4$ y \pm 1 y. Healthy adult control patients were also used as methodologic controls for both surface and cytoplasmic Ig-expressing B-lymphocytes. This protocol was reviewed and approved by The Children's Memorial Institute for Education and Research Review Board. Parental consent was obtained for subjects and controls.

All KD patients had pre- and post-IVGG blood samples collected. Pre-IVGG samples were collected on median $\mathrm{d} 7$ after onset of fever (range $=5-10 \mathrm{~d}$ ). Post-IVGG samples were collected at the first follow-up visit $(n=15$, median illness day $=15$, range $=14-21 \mathrm{~d}$ ). Eight of the 15 patients had additional samples taken at their second follow-up visit (median illness day $=55$, range $=41-70 \mathrm{~d}$ ). Only one patient was found to have significant echocardiographic abnormalities, with dilatation of the left main and left anterior descending arteries noted before and after IVGG treatment.

The febrile control group consisted of 10 children (4 males, 6 females). The median age was 65 mo with a range of 26-102 mo. Diagnoses in these patients included pneumonia $(n=3)$, urinary tract infection $(n=2)$, empyema $(n=2)$, bacterial meningitis $(n=1)$, toxic shock syndrome $(n=1)$, and viral myocarditis $(n=1)$.

The nonfebrile control group consisted of 15 children (7 males, 8 females). The median age was 47 mo with a range of 6-105 mo. Reasons for elective surgery included tonsillectomy/adenoidectomy $(n=8)$, hernia repair $(n=6)$, and dental extraction $(n=1)$.

The adult control group consisted of 14 healthy adults (5 males, 9 females).

\section{Reagents}

Fluorochrome-conjugated murine MAb to the following human antigens were purchased from Becton Dickinson Biosciences (San Jose, CA, U.S.A.): CD20, CD38, CD3, CD19, CD16+56, and CD45. Fluorochrome-conjugated and unconjugated goat MAb to human $\operatorname{IgA}, \operatorname{IgM}$, IgD, and IgG with isotype control were purchased from Caltag Laboratories (Burlingame, CA, U.S.A.). Unconjugated antibodies (IgA, IgM, $\mathrm{IgD}$, and $\mathrm{IgG}$ ) were used to block surface immunoglobulin during the intracytoplasmic immunoglobulin staining process.

\section{Immunophenotyping}

Surface immunoglobulin staining. Peripheral blood lymphocyte subset immunophenotyping was performed using standard whole blood lysis methodology (9). Briefly, $100 \mu \mathrm{L}$ of EDTA anticoagulated whole blood was added to tubes containing three MAb each conjugated with a different fluorochrome (see Table 1). After a 15-min incubation period in the dark at room temperature, the red blood cells were lysed; the remaining cells were washed and then fixed in $1 \%$ paraformaldehyde and stored at $4{ }^{\circ} \mathrm{C}$ until analyzed by flow cytometry.

Intracellular immunoglobulin staining. Staining for intracellular immunoglobulin was performed as described by Schmid et al. (10) using commercially available fixation and permeabilization reagents (an der Grub Fix\&Perm kit, Scandic $\mathrm{GmbH}$, Vienna, Austria). Cells are first stained for surface

Table 1. Monoclonal antibody panels (Becton Dickinson Biosciences, San Jose, CA) used to identify major lymphocyte subsets and proportion of B-lymphocytes expressing surface immunoglobulin $A, M, D$, and $G$

\begin{tabular}{llll}
\hline & \multicolumn{1}{c}{ FITC } & \multicolumn{1}{c}{ Phycoerythrin (PE) } & \multicolumn{1}{c}{$\begin{array}{c}\text { Peridinin chlorophyll } \\
\text { protein (PerCP) }\end{array}$} \\
\hline Monoclonal antibody & CD3 & CD19 & CD45 \\
Subset & Pan T-lymphocytes & B-lymphocytes & Lymphocyte gating reagent \\
Monoclonal antibody & CD3 & CD16+56 & CD45 \\
Subset & Pan T-lymphocytes & NK cells & Lymphocyte gating reagent \\
Monoclonal antibody & IgA or IgM or IgD or IgG & CD38 & CD20 \\
Subset & Surface Ig & Plasma cells very bright CD38 & Pan B-lymphocytes \\
\hline
\end{tabular}


expressing molecules with CD20-PerCP and CD38-PE (Becton Dickinson Biosciences) in addition to purified anti-IgA, $\operatorname{IgM}, \operatorname{IgD}$, or $\operatorname{IgG}$ (i.e. not fluoresceinated) as above. The purified, unlabeled antibodies are used to block surface immunoglobulin from binding FITC-labeled anti-immunoglobulin antibody used to detect cytoplasmic immunoglobulin in permeabilized cells. The membrane labeled cells are then added to fixation medium for $15 \mathrm{~min}$ in the dark at room temperature, after which they are washed, and the supernatants removed. The pellet is resuspended and $100 \mu$ of the permeabilization medium and $20 \mu \mathrm{L}$ of FITC-conjugated anti-IgA, IgM, IgG, $\mathrm{IgD}$, or isotype control (Caltag Laboratories) are added, the sample is incubated at $4{ }^{\circ} \mathrm{C}$ for $20 \mathrm{~min}$. The samples are then washed twice and resuspended in PBS containing $0.1 \% \mathrm{NaN}_{3}$ and $5 \%$ fetal bovine serum. Samples are immediately analyzed on the flow cytometer.

\section{Flow Cytometric Analysis}

Three-color flow cytometry acquisition and analysis was performed on a Becton Dickinson FACScan flow cytometer. Acquisition was performed with Lysis II software and analysis was performed with Cellquest (BD Biosciences, San Jose, CA, U.S.A.). The cytometer was calibrated daily for sensitivity, alignment, and spectral overlap using the standard laboratory practice as defined by the manufacturer (FACScomp software with CaliBrite beads, BD Biosciences, San Jose, CA, U.S.A.). For every sample, 10,000 lymphocyte gated events (identified as a cluster with specific right-angle light scatter characteristics and specific levels of CD45 expression) were acquired and stored as list mode files. For analysis of the expression of surface markers (i.e. lymphocyte subset immunophenotyping on whole blood samples), results were expressed as the percentage of lymphocytes expressing specific subset-defining markers. Positive expression was identified as fluorescence above the background as determined by isotype- and fluorochrome-matched nonleukocyte specified MAb (i.e. negative control). Sample integrity and quality was ascertained by the following criteria: the sum of the B-lymphocyte, Tlymphocyte, and NK cell percentages had to be $100 \pm 10 \%$ and the reproducibility of the percentage of T-lymphocytes within duplicate tubes had to be $\leq 4 \%$. Samples that did not fulfill these criteria were discarded and repeated where possible.

The percentage of B-lymphocytes expressing IgA, IgM, IgD, or IgG was assessed by drawing an analysis gate (Fig. 1) around cells expressing lymphocyte right-angle light scatter characteristics and either surface CD20 (B-lymphocytes) or bright surface CD38 (differentiated B-lymphocytes/plasma cells). For intracellular immunoglobulin expression, the same analytical procedure was performed. $\mathrm{IgD}$ served as a positive control for the intracellular assay and a nonlymphocytespecific isotype control antibody served as the negative control as well as the positive-negative fluorescence discriminator. All antibodies used for intracellular staining were $\mathrm{F}\left(\mathrm{ab}^{\prime}\right) 2$ antibody preparations.

\section{Statistical Analysis}

Values for the index case at presentation were compared with each of the following: his or her own sample at 2 and $8 \mathrm{wk}$ after IVGG, a nonfebrile control, a febrile control, and a healthy adult. Nonparametric methods of analysis (Wilcoxon rank sum and signed-rank test) and median values were used because of small sample size and asymmetric distribution of parameters. For each parameter, pre-IVGG samples were compared with each of the convalescent and control samples. A result was regarded as significant if the two-sided $p$ value was $<0.05$.

\section{RESULTS}

Fifteen patients with acute KD were enrolled in this study, including 7 males and 8 females. The median age was $59 \mathrm{mo}$, with a range from 9 to 92 mo. The absolute number of T-lymphocytes $(\mathrm{CD} 3+$ lymphocytes) in the samples obtained from acute KD patients (pre-IVGG) was significantly lower compared with the samples obtained from the first and second convalescent visits ( $p=0.02$ and 0.01 , respectively; Wilcoxon signed-rank test). The absolute number of T-lymphocytes was also lower in the acute KD specimens compared with pediatric controls (febrile controls $p=0.04$, afebrile controls $p<0.001$; Wilcoxon rank sum test) (Table 2). The absolute number of NK cells (CD16/56 + lymphocytes) was significantly lower in pre-IVGG samples compared with convalescent samples $(p=$ 0.005 and $p=0.01$, respectively) and afebrile controls ( $p=$ $0.001)$ but not compared with other control groups. No significant changes were observed in absolute numbers of Blymphocytes (CD19+ lymphocytes) among the study groups.

The absolute number of circulating surface IgA-positive B-lymphocytes $(\operatorname{IgA}+, \mathrm{CD} 20+)$ was significantly lower in acute KD patients compared with pediatric and adult control groups (febrile controls $p=0.01$, afebrile controls $p<0.001$, and adult controls $p=0.03$, summarized in Table 3 ). The absolute number of cytoplasmic IgA B-lymphocytes (IgA+, CD20+) was significantly lower in pre-IVGG samples compared with the second convalescent samples $(p=0.04)$ and to controls (febrile controls $p=0.003$, afebrile controls $p<$ 0.001 , and adult controls $p<0.001$ ). The absolute number of surface $\operatorname{IgA}+$ differentiated B-lymphocytes ( $\operatorname{IgA}+$, bright CD38+) was significantly lower in pre-IVGG samples compared with febrile and afebrile control groups $(p=0.008$ and $p=0.004$, respectively). No differences were observed among all $\mathrm{KD}$ and control groups for cytoplasmic $\mathrm{IgA}+$ differentiated B-lymphocytes (IgA+, bright CD38+). Further analysis of absolute numbers of IgA-expressing cells did not reveal a relationship with the time of sampling (i.e. d 5 to d 10), however, this analysis was limited by the small number of samples (data not shown). Analysis of control groups did not show any specific outliers, particularly in the febrile group where a variety of different febrile illness, including one patient with toxic shock syndrome, had been included.

The absolute number of surface $\mathrm{IgG}+\mathrm{B}$-lymphocytes $(\mathrm{IgG}+$, $\mathrm{CD} 20+)$ and $\mathrm{IgG}+$-differentiated B-lymphocytes $(\mathrm{IgG}+$, bright $\mathrm{CD} 38+$ ) was not significantly different among all $\mathrm{KD}$ samples and all control groups (summarized in Table 4). The absolute 


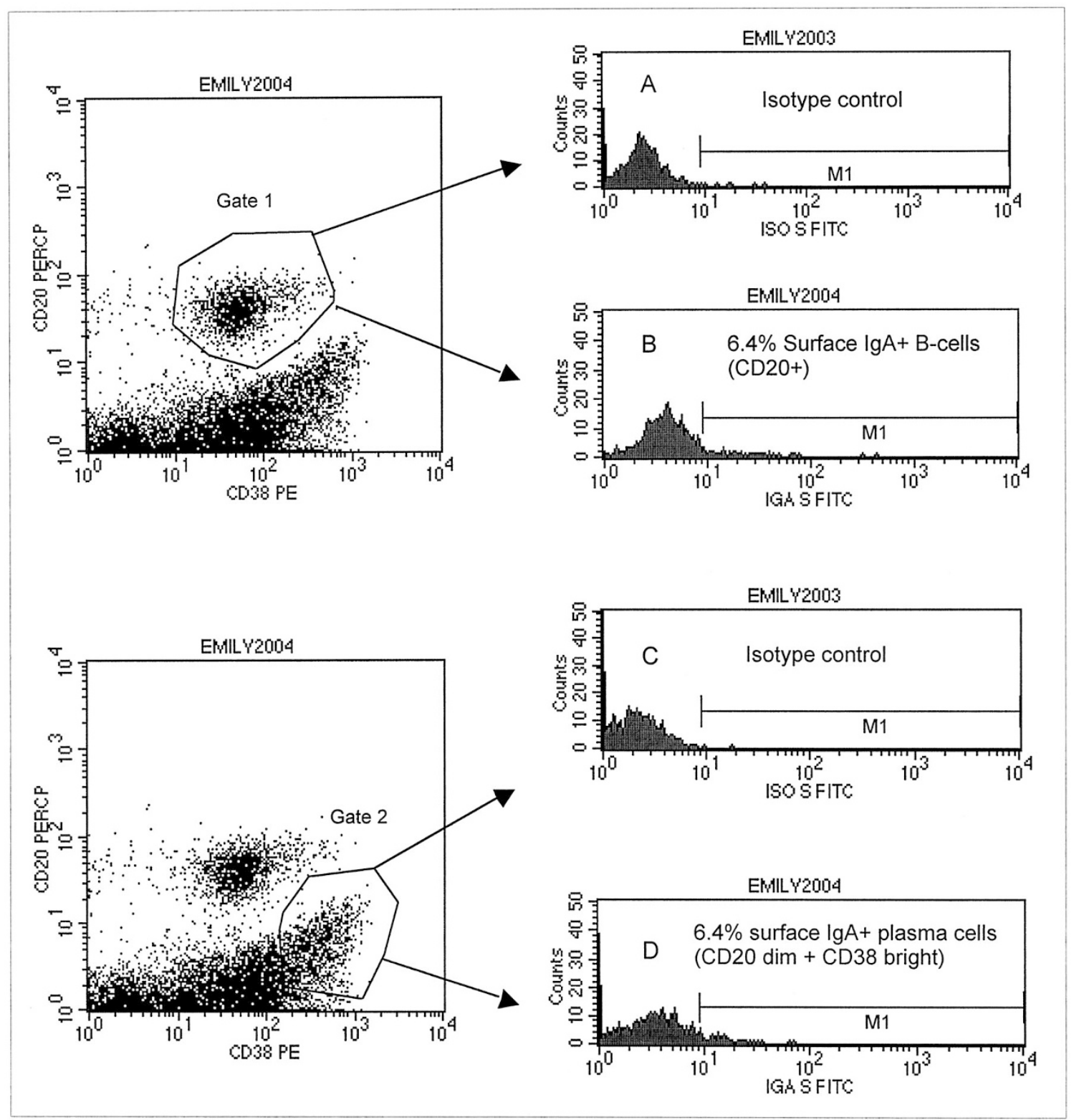

Figure 1. Representative dot plots and histograms showing gating strategy for identifying B-lymphocytes (CD20+ lymphocytes) and plasma cells (CD38+bright lymphocytes). Gate 1 identifies CD20 bright lymphocytes and Gate 2 identifies CD20 dim + CD38 bright lymphocytes. $A$ and $C$ show isotype control histograms where the marker (M1) is set to determine the cut-off for positive expression. $B$ and $D$ show surface IgA histograms (CD20 bright and CD38 bright, respectively) with positive expression represented as a percentage above background fluorescence.

Table 2. Median absolute numbers $\left(\times 10^{9} / \mathrm{L}\right)$ of lymphocyte subsets in patients with Kawasaki disease and controls

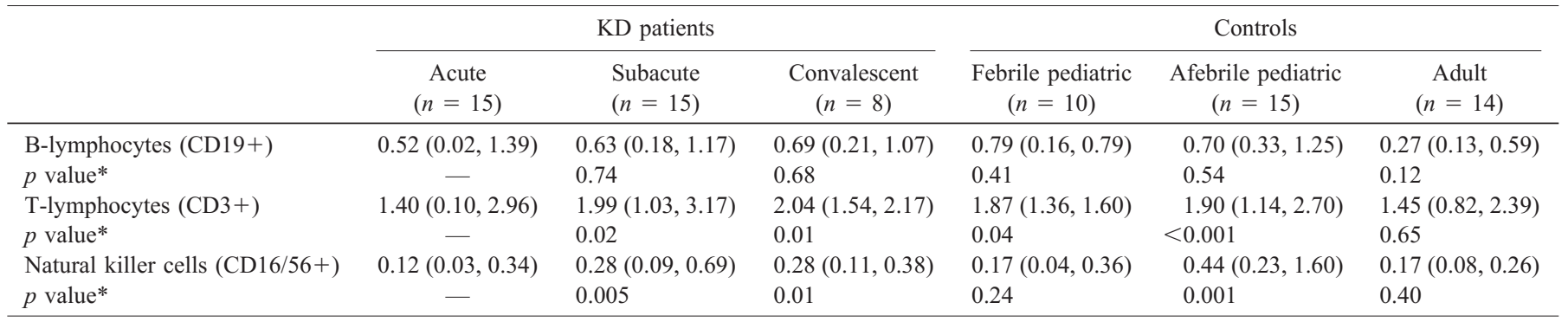

Values expressed as median (5th, 95th centiles).

* Comparison with acute KD sample using Wilcoxon signed-rank and rank sum tests.

number of cytoplasmic IgG+ B-lymphocytes $(\mathrm{IgG}+, \mathrm{CD} 20+)$ was significantly lower in the acute KD patients (pre-IVGG samples) compared with febrile controls $(p=0.02)$, afebrile controls $(p=0.001)$, and adult controls $(p=0.01)$. Similarly, the absolute number of circulating cytoplasmic $\mathrm{IgG}+$-differentiated B-lymphocytes (IgG+, bright $\mathrm{CD} 38+$ ) was significantly lower in 
Table 3. Median absolute numbers $\left(\times 10^{9} / \mathrm{L}\right)$ of surface and cytoplasmic IgA B-lymphocytes $\left(\operatorname{Ig} A^{+}, \operatorname{CD}^{+} 0^{+}\right)$and $\operatorname{IgA}$ plasma cells $\left(\operatorname{Ig} A^{+}\right.$, bright $\mathrm{CD}_{38^{+}}$) in patients with Kawasaki disease and controls

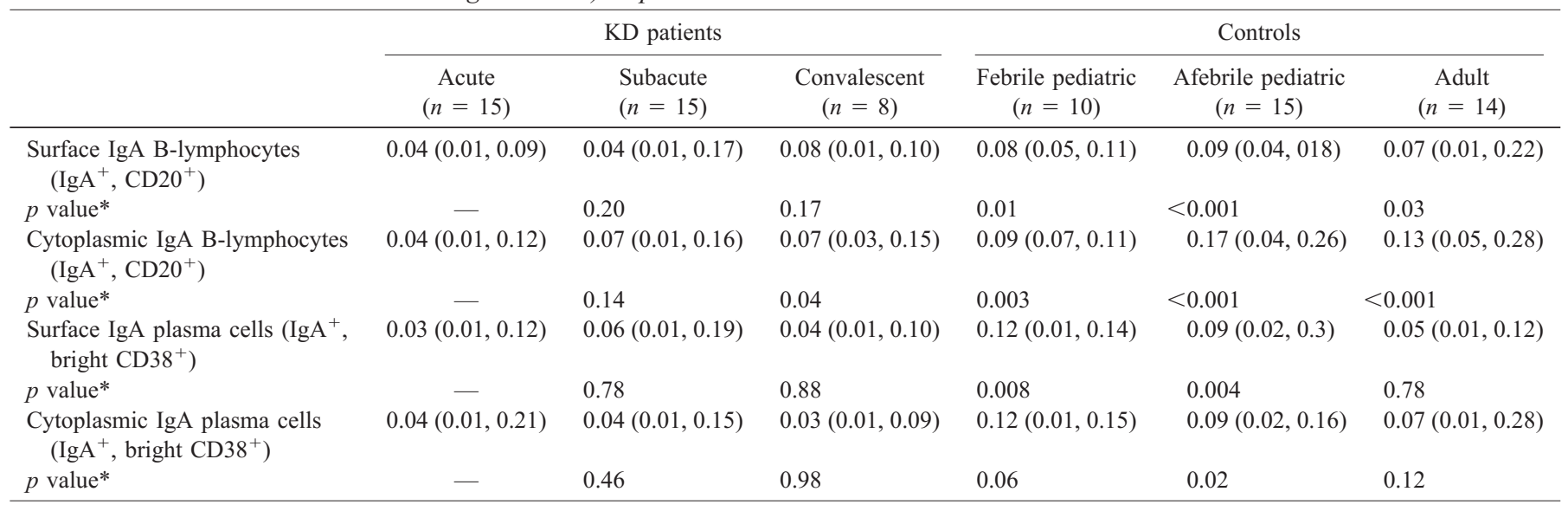

Values expressed as median (5th, 95th centiles).

* Comparison with acute KD sample using Wilcoxon signed-rank and rank sum tests.

Table 4. Median absolute numbers $\left(\times 10^{9} / \mathrm{L}\right)$ of surface of cytoplasmic $\operatorname{IgG~B-lymphocytes}\left(\operatorname{IgG}{ }^{+}, \mathrm{CD}^{2} 0^{+}\right)$and $\operatorname{IgG}$ plasma cells $\left(\operatorname{Ig} \mathrm{G}^{+}\right.$, bright $\left.C D 38^{+}\right)$in patients with Kawasaki disease and controls

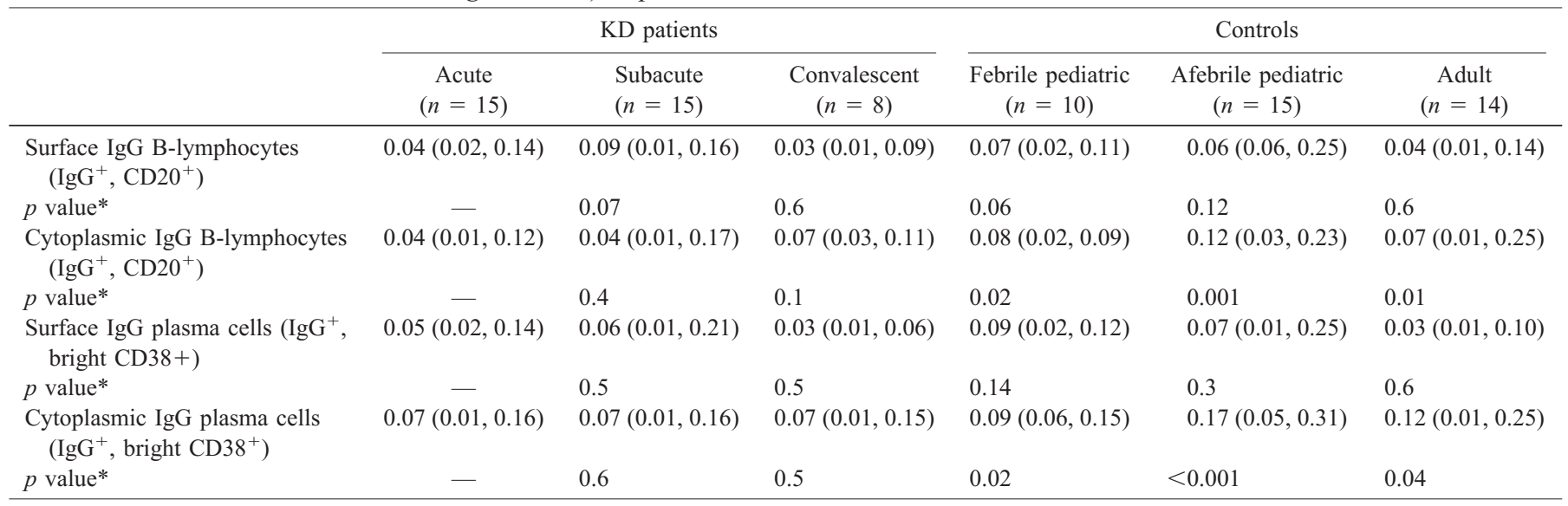

Values expressed as median (5th, 95th centiles).

* Comparison with acute KD sample using Wilcoxon signed-rank and rank sum tests.

the acute KD patients (pre-IVGG samples) compared with the febrile controls $(p=0.02)$, afebrile controls $(p<0.001)$, and adult controls $(p=0.04)$. No significant differences between the acute $\mathrm{KD}$ patients and controls were noted with respect to absolute numbers of circulating surface $\mathrm{IgD}+$-differentiated $\mathrm{B}$ lymphocytes $(\operatorname{IgD}+$, bright $\mathrm{CD} 38+)$, surface $\operatorname{IgD}+\mathrm{B}$ lymphocytes (IgD+, $\mathrm{CD} 20+)$, cytoplasmic $\operatorname{IgD}+$ B-lymphocytes (IgD+, CD20+), and cytoplasmic IgD+differentiated B-lymphocytes (IgD+, bright CD38+). Similarly, no differences were noted between the KD patients and control groups with respect to surface IgM+ B-lymphocytes (IgM+, $\mathrm{CD} 20+$ ), surface IgM+-differentiated B-lymphocytes (IgM+, bright $\mathrm{CD} 38+$ ), cytoplasmic IgM + B-lymphocytes (IgM+, $\mathrm{CD} 20+$ ), and cytoplasmic IgM+-differentiated B-lymphocytes $(\operatorname{IgM}+$, bright $\mathrm{CD} 38+)$ (data not shown).

\section{DISCUSSION}

We did not find an increase in absolute numbers of circulating IgA-expressing cells in acute $\mathrm{KD}$, but did, surprisingly, find a selective reduction of these cells compared with healthy and febrile children and healthy adults. No differences in absolute numbers of circulating surface IgM-expressing Blymphocytes were observed in any of the $\mathrm{KD}$ and control groups. Because IgA plasma cells infiltrate coronary artery, upper respiratory tract, kidney, and pancreas in acute KD (6), the finding of selectively decreased numbers of these cells in the peripheral blood in acute KD was unexpected.

A significant reduction in the absolute numbers of circulating T-lymphocytes (CD3+ lymphocytes) and NK cells (CD16/56+ lymphocytes) was observed during the acute phase of $\mathrm{KD}$ followed by an increase after IVGG therapy, similar to previously reported findings (11-15). No significant differences were observed in absolute circulating B-lymphocyte (CD19+ lymphocytes) numbers among all $\mathrm{KD}$ and control samples. Although some studies have demonstrated alterations in B-lymphocyte percentages in acute $\operatorname{KD}(13,16,17)$, our study is in agreement with the recent study of Choi and colleagues (18), showing no changes in circulating absolute B-lymphocyte numbers in acute KD. Reports of B-lymphocyte numbers in acute KD may vary in different studies because of differences in timing of blood sampling by illness day, and use of absolute or relative size of lymphocyte populations. 
Early studies investigating the humoral response in acute $\mathrm{KD}$ peripheral blood have reported polyclonally increased serum immunoglobulin (IgA, IgM, IgG, and IgE) during the first month of illness (19), increased spontaneous immunoglobulin production by B-lymphocytes $(20,21)$, and reversal of these findings following IVGG therapy (22). Increased levels of serum IgA-, IgG-, and IgE-containing circulating immune complexes (CIC) have also been found during the acute phase of $\mathrm{KD}(19,23,24)$, with up to $68 \%$ of CIC containing IgG and $85 \%$ containing IgA (25). Ohshio and colleagues similarly demonstrated elevated levels of IgA CIC with higher levels at $11-23 \mathrm{~d}$ of illness compared with $<10 \mathrm{~d}$ of illness (26).

IgA plasma cell infiltration has been reported in KD target tissues, particularly coronary arteries (4). IgA plasma cells were also demonstrated in aortic and renal artery vascular tissue, suggesting the importance of these cells in systemic KD-related vasculitis. Subsequent analysis of nonvascular tissue, such as the respiratory tract, pancreas and kidney, has shown a similar IgA plasma cell infiltrate independent of the presence of vasculitis in the respective organ (6). Of note, there was a remarkable increase in IgA plasma cells in the upper respiratory tract of patients with fatal $\mathrm{KD}$, similar to that seen in fatal respiratory viral infection. These unique findings, in combination with the epidemiologic features of $\mathrm{KD}$, support a respiratory and/or gastrointestinal portal of entry of the KD etiologic agent(s).

A potential limitation of our study is that KD patients were treated with IVGG before the 10th illness day, and it is possible that IVGG may have a direct effect on IgA B-lymphocyte populations in the subacute and convalescent stages separate from the disease process itself. However, it seems unlikely that IVGG would result in a selective decrease in B-lymphocyte numbers of the $\operatorname{IgA}$ isotype but not of the other isotypes.

The finding that circulating IgA B-lymphocyte numbers were lower in acute $\mathrm{KD}$ patients than in all control groups is intriguing. Decreased numbers of circulating IgA Blymphocytes could be the result of their decreased release into the peripheral blood from lymphoid tissues and bone marrow, increased cell turnover, or enhanced migration out of the peripheral blood into tissues. In view of the infiltration of IgA plasma cells into vascular tissue, respiratory tract, kidney, and pancreas that we have observed in acute KD (6), decreased production or release into peripheral blood seems unlikely, as does an increase in cell turnover or death. The finding of decreased numbers of circulating surface IgA B-lymphocytes in acute KD appears most consistent with the hypothesis that these cells exit the peripheral blood and migrate to selective KD tissues, particularly arterial walls, where they undergo terminal differentiation into plasma cells. We speculate that IgA B-lymphocytes are selectively withdrawn from the peripheral circulation into target tissues as part of a specific IgA response in KD. Further studies of lymphocyte trafficking and homing in acute KD appear warranted.

In conclusion, we have demonstrated that circulating IgA B-lymphocytes and plasma cells are reduced in acute KD. This finding complements the novel finding of IgA plasma cell infiltration into selective KD tissues, particularly arterial walls, during acute $\mathrm{KD}$, and suggests that these cells play an important role in the pathogenesis of the illness.

Acknowledgment. The authors thank Nancy Innocentini for help with sample and data collection.

\section{REFERENCES}

1. Shulman ST, DeInocencio J, Hirsh R 1995 Kawasaki disease. Pediatr Clin North Am 42:1205-1222

2. Kato H, Inchinose E, Yoshioka F, Takecki T, Matsunaga S, Suzuki K, Rikitake N 1982 Fate of coronary aneurysm in Kawasaki Disease; serial coronary angiography and long-term follow up study. Am J Cardiol 49:1758-1766

3. Suzuki A, Kamiya T, Kuwahara N, Ono Y, Kohata T, Takahashi O, Kimura K, Takayima M 1996 Coronary arterial lesions of Kawasaki Disease: cardiac catheterization findings in 1100 cases. Pediatr Cardiol 7:3-9

4. Rowley AH, Eckerly C, Jack H, Shulman S, Baker S 1997 IgA plasma cells in vascular tissue of patients of patients with Kawasaki Syndrome. J Immunol 159:5946-5955

5. Rowley AH, Shulman ST, Spike BT, Mask CA, Baker SC 2001 Oligoclonal IgA Response in the vascular wall in acute Kawasaki disease. J Immunol 166:1334-1343

6. Rowley AH, Shulman ST, Mask CA, Finn LS, Terai M, Baker SC, Galliani CA, Takahashi K, Naoe S, Kalelkar MB, Crawford SE 2000 IgA plasma cell infiltration of proximal respiratory tract, pancreas, kidney and coronary artery in acute Kawasaki disease. J Infect Dis 182:1183-1191

7. Wedgewood J, Weinberger B, Hatam L, Palmer R 1991 Umbilical cord blood lacks circulating B lymphocytes expressing surface IgG and IgA. Clin Immunol Immunopathol 84:276-282

8. Harada Y, Michio M, Huang N, Mahmoud M, Lisukov I, Mihara K, Tsujimoto T, Kuramoto A 1996 Identification of early plasma cells in peripheral blood and their clinical significance. Br J Haematol 92:184-191

9. Jackson A, Warner N 1986 Preparation, staining and analysis by flow cytometry of peripheral blood leukocytes. In: Rose N, Friedman H, Fahey J (eds) Manual of Clinical Laboratory Immunology, Washington, DC, American Society of Microbiology, pp 226-235

10. Schmid I, Uittenbogaart C, Giorgi J 1991 A gentle fixation and permeabilization method for combined cell surface and intracellular staining with improved precision in DNA quantification. Cytometry 12:279-285

11. Terai M, Kohno Y, Niwa K, Toba T, Sakurai N, Nakajima H 1987 Imbalance among T-cell subsets in patients with coronary arterial aneurysm in Kawasaki disease. Am J Cardiol 60:555-559

12. Furukawa S, Matsubara K, Tsuji K, Okumara K, Yabuta K 1993 Transient depletion of $\mathrm{T}$ cells with bright $\mathrm{CD} 11 \mathrm{a} / \mathrm{CD} 18$ expression from peripheral circulation during acute Kawasaki disease. Scand J Immunol 37:377-380

13. Jason J, Gregg L, Han A, Hu A, Inge K, Eick A, Tham I, Campbell R 1997 Immunoregulatory changes in Kawasaki disease. Clin Immunol Immunopathol 84:296-306

14. Furukawa S, Matsubara K, Tsuji K, Motohashi T, Watanabe T, Umezawa Y, Yabuta K 1991 Comparison of Kawasaki disease and infectious mononucleosis in terms of natural killer cells and CD8 + T cell subsets. J Infect Dis 163:416-417

15. Finberg RW, Newburger J, Mikati M, Heller A, Burns J 1992 Effect of high doses of intravenously administered immune globulin on natural killer cell activity in peripheral blood. J Pediatr 120:376-380

16. Furukawa S, Matsubara T, Yabuta K 1992 Mononuclear cell subsets and coronary artery lesions in Kawasaki disease. Arch Dis Child 67:706-708

17. Saulsbury FT 1992 The effect of intravenous immunoglobulin on lymphocyte populations in children with Kawasaki syndrome. Clin Exp Rheumatol 10:617-620

18. Choi I-H, Chwae Y, Shim W, Kim D, Kwon D, Kim J 1997 Clonal expansion of CD8 + T cells in Kawasaki disease. J Immunol 159:481-486

19. Lin C-Y, Hwang B 1987 Serial immunologic studies in patients with mucocutaneous lymph node syndrome (Kawasaki disease). Ann Allergy Asthma Immunol 59:291297

20. Leung DY, Chu E, Wood N, Grady S, Meade R, Geha R 1983 Immunoregulatory T cell abnormalities in mucocutaneous lymph node syndrome. J Immunol 130:2002 2004

21. Leung DY, Siegel R, Grady S, Krensky A, Meade R, Reinherz E, Geha R 1982 Immunoregulatory abnormalities in mucocutaneous lymph node syndrome. Clin Immunol Immunopathol 23:100-112

22. Leung DY, Burns J, Newburger J, Geha R 1987 Reversal of lymphocyte activation in vivo in Kawasaki Syndrome by intravenous gammmaglobulin. J Clin Invest 79:468472

23. Mason W, Jordan S, Sakai R, Takahashi M 1988 Lack of effect of gamma-globulin infusion on circulating immune complexes in patients with Kawasaki syndrome. Ped Infect Dis J 7:94-99

24. Salo E, Kekomaki R, Pelkonen P, Viander M, Wagner O 1988 Kawasaki disease: monitoring of circulating immune complexes. Eur J Pediatr 147:377-380

25. Levin M, Holland P, Nokes T, Novelli V, Mola M, Levinsky R, Dillon M, Barrat T, Marshall W 1985 Platelet immune complex interaction in pathogenesis of Kawasaki disease and childhood polyarteritis. BMJ 290:1456-1460

26. Ohshio G, Furukawa F, Khine M, Yoshioka H, Kudo H, Hamashima Y 1987 High levels of IgA-containing circulating immune complex and secretory IgA in Kawasaki disease. Microbiol Immunol 31:891-898 